\title{
Parameter Estimation of a Delay Time Model of Wearing Parts Based on Objective Data
}

\author{
Y. Tang, ${ }^{1}$ J. J. Jing, ${ }^{2}$ Y. Yang, ${ }^{3}$ and C. Xie ${ }^{1}$ \\ ${ }^{1}$ School of Mechatronic Engineering, Southwest Petroleum University, Chengdu 610500, China \\ ${ }^{2}$ Safety, Environment, Quality Supervision \& Testing Research Institute, CCDE, Guanghan 618000, China \\ ${ }^{3}$ School of Science, Southwest Petroleum University, Chengdu 610500, China \\ Correspondence should be addressed to Y. Tang; tangyanggreat@163.com
}

Received 10 June 2014; Revised 22 September 2014; Accepted 27 October 2014

Academic Editor: Wenbin Wang

Copyright (c) 2015 Y. Tang et al. This is an open access article distributed under the Creative Commons Attribution License, which permits unrestricted use, distribution, and reproduction in any medium, provided the original work is properly cited.

\begin{abstract}
The wearing parts of a system have a very high failure frequency, making it necessary to carry out continual functional inspections and maintenance to protect the system from unscheduled downtime. This allows for the collection of a large amount of maintenance data. Taking the unique characteristics of the wearing parts into consideration, we establish their respective delay time models in ideal inspection cases and nonideal inspection cases. The model parameters are estimated entirely using the collected maintenance data. Then, a likelihood function of all renewal events is derived based on their occurring probability functions, and the model parameters are calculated with the maximum likelihood function method, which is solved by the CRM. Finally, using two wearing parts from the oil and gas drilling industry as examples - the filter element and the blowout preventer rubber core-the parameters of the distribution function of the initial failure time and the delay time for each example are estimated, and their distribution functions are obtained. Such parameter estimation based on objective data will contribute to the optimization of the reasonable function inspection interval and will also provide some theoretical models to support the integrity management of equipment or systems.
\end{abstract}

\section{Introduction}

For the reliability maintenance and security management of equipment, the establishment of the failure time distribution model (FTDM) of parts or systems is one of the key steps toward making scientific maintenance decisions [1]. Parameter estimation is the first step in the application of the FTDM, and the accuracy and precision of these estimations directly affects whether the optimized predetermined maintenance interval is reasonable. Therefore, parameter estimation is a crucial part in the process of the establishment of the FTDM. When a substantial amount of maintenance data can be collected, the parameter estimation method can use completely objective data, which is more accurate than subjective data [2]. The failure frequency of wearing parts is relatively higher than other parts used in the operation process of the equipment. Traditional solutions to ensure the safe and reliable operation of the equipment include initiating functional inspections and repairs/replacements based on the experience and subjective judgment of personnel. Since the maintenance personnel do not identify their failure time distribution rule by the quantitative method, they will develop irrational function inspection intervals, which may lead to excess maintenance, a reduction in the reliability of the system, lower production efficiency, and so on. Before the occurrence of the functional failure of the wearing parts, we found that some symptoms were often present, such as fatigue cracks, wear, and corrosion. These symptoms are a potential failure process of the wearing parts. Though they present no immediate threat to the equipment, if, allowed to accumulate for a period of time, such defects will gradually increase and reveal themselves and cause the functional failure of the equipment. This failure process is consistent with the delay time theory.

In 1973, Christer put forward the concept of the delay time model (DTM) in the context of building maintenance [3]. 
A number of research papers followed, applying the DTM to industrial asset inspection problems. Christer and Waller [4] used subjective data to solve the DTM parameters, and Christer and Redmond [5] studied the maximum likelihood function method of the subjective estimate of the DTM. Corresponding with the subjective estimation methods, Baker and Wang $[6,7]$ put forward a parameter estimation method founded on objective data. Christer and Wang [8] then proposed a method that uses subjective and objective data to solve the DTM for multicomponents in complex systems. Recent research on the DTM includes the work by Wang et al. [9], who proposed a parameter estimation method of the maximum likelihood of the DTM, and $\mathrm{Hu}$ et al. [10], who established a nonideal DTM solved by a genetic algorithm. Wang $[11,12]$ also presented a two-stage prognosis model in condition-based maintenance and an inspection model based on a three-stage failure process.

However, in these previous studies, the parameter estimation of the DTM was solved by using simulation data, subjective data, or a combination of subjective data and objective data, mainly because there was rarely sufficient maintenance data to allow the use of fully objective data to solve it [1316]. Moreover, the formula of the likelihood function of the DTM is quite complex because of both the failure density function and the failure probability function with the integral term, and the maximum could not be solved by traditional derivation methods $[17,18]$. However, we found that, from the process of frequent functional inspections and maintenance renewals of the wearing parts, a large amount of maintenance data is collected in a short period of time. The parameters of the established DTM of the wearing parts could therefore be estimated based on sufficient objective data. This effectively removes the subjective factors and reduces the estimation error of the parameters. It is necessary to explore a novel algorithm to effectively look for the complicated maximum likelihood function of the DTM and to get the parameter estimation values. Thus, we will obtain the FTDM of the wearing parts, which can help to optimize the maintenance interval and make scientific maintenance decisions in the next study.

The remainder of this paper is organized as follows. In Section 2, we introduce the concept of the function inspection and the DTM. In Section 3, we establish the ideal inspection model and the nonideal inspection model of the wearing parts. Section 4 is devoted to estimating the parameters of the DTM by maximizing the log-likelihood, which is solved with the CRM. In Section 5, we take two examples from the oil and gas drilling industry to verify the DTM of the wearing parts and the solving method. We provide a brief discussion of our results in Section 6.

\section{The Delay Time Concept}

2.1. Function Inspection. A schematic diagram of function inspection for the parts or components is illustrated in Figure 1 [19]. The function inspection is carried out at the time "Point A" and the inspection is perfect, so "Defect 1" can be identified and the parts will be repaired and replaced. Similarly, if a perfect function inspection is carried out at

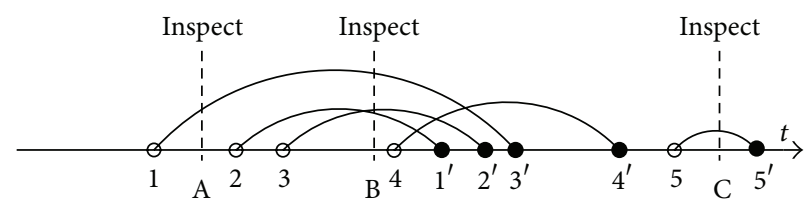

Figure 1: Function inspection schematic diagram, where " $O$ " denotes the initial defect point of a part; "" denotes failure points caused by a defect; and A, B, and C denote the times of the three function inspections.

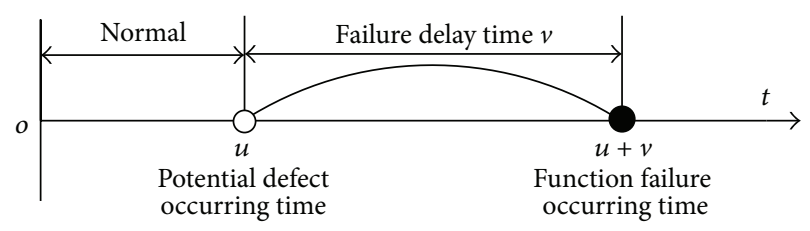

FIgURE 2: The DTM schematic diagram.

the time "Point B", the failures at times $1^{\prime}, 2^{\prime}$ and $3^{\prime}$ can be avoided. If the inspection is carried out at the time "Point $C$ ", then "Defect 5 " can be identified prior to failure, but failures at times $1^{\prime}, 2^{\prime}, 3^{\prime}$ and $4^{\prime}$ will have already occurred. In summary, from the function inspection of the three different times above, we know that a fault could be averted only if the inspection is set between the defect's appearance time and the failure occurrence time. Therefore, it is necessary to determine a reasonable regular inspection to lower the failure rate of parts.

2.2. Delay Time Concept. The delay time concept is similar in definition to the well-known potential failure interval in reliability-centered maintenance (RCM), defining the failure process of an asset as a two-stage process, as shown in Figure 2. The first stage is the normal operating stage of an asset, from new to the point that a potential defect is identified. The second stage is defined as the delay time, which is from the point of the potential defect to the point of function failure [2]. Through research and analysis, the most wearing parts (e.g., a filter core, bop rubber core, mud pump bearings, etc.) comply with the DTM. It is the existence of such a delay time that provides the opportunity for preventive maintenance (PM) to be carried out, to remove the identified defects before the parts fail. With appropriate modeling of the durations of these two stages, optimal function inspection intervals can be identified to optimize a criterion function of interest.

\section{The DTM of Wearing Parts}

\subsection{Notation and Assumptions}

3.1.1. Notation. In this paper, we use the following notation. $u$ : The initial time of the defect $g(u)$ : Probability density function of the initial time $u$ $G(u)$ : Cumulative distribution function of the initial time $u$ 
$v$ : The delay time of the defect

$f(v)$ : Probability density function of the delay time $v$ $F(v)$ : Cumulative distribution function of the delay time $v$

$N_{I}$ : The total number of inspection renewals

$N_{F}$ : The total number of failure renewals

$P_{m}\left(t_{i}\right)$ : The probability of the $i$ th inspection renewal happening at time $t_{i}$

$p_{b}\left(t_{j}\right)$ : The density function of the $j$ th failure renewal happening at time $t_{j}$

$P_{n}(t)$ : The probability of no failure happening during the whole of observation period $t$

$t_{i}$ : The occurrence time of the $i$ th inspection renewal

$t_{m}$ : The last inspection time before the $i$ th inspection renewal

$t_{j}$ : The occurrence time of the $j$ th inspection renewal $t_{n}$ : The last inspection time before the $j$ th inspection renewal

$t_{k}$ : The last inspection time before the end of the observation

$t$ : The time of end of the observation

$m$ : The total inspection number before $i$ th times inspection renewal

$n$ : The total inspection number before $j$ th times failure renewal

$k$ : The total inspection number before the end of the observation

$t_{c}$ : The $c$ th opportunity renewal time

$N_{c}$ : The total number of opportunity renewals.

3.1.2. Assumptions. The following assumptions are used in this paper.

(1) The wearing part is a single component, inspected at time point $t_{i}\left(t_{i}=i T, i=1,2,3, \ldots\right)$, where $T$ is a regular inspection interval.

(2) When defects are identified at the inspection point, they need to be repaired immediately. Otherwise, the wearing part would continue to work until either a failure occurs or the next inspection is reached.

(3) If defects are identified or function failures occur between two continual inspection points, the wearing part needs be immediately repaired or replaced.

3.2. The Ideal Inspection Model. The defects can easily be found for some wearing parts due to their simple structures. We have assumed that the process of the function inspection for these kinds of wearing parts is an ideal case. We can detect any defects for such wearing parts perfectly at the next inspection time point. There are three kinds of events in the failure process for the wearing parts: a failure renewal event,

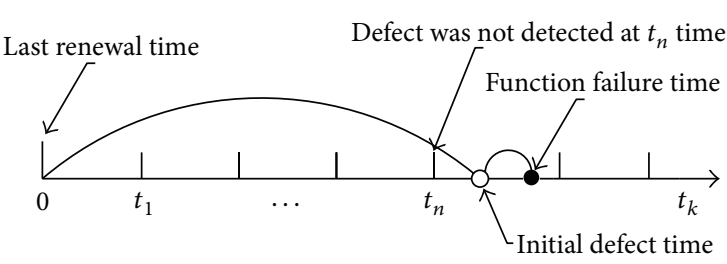

(a) Failure renewal event

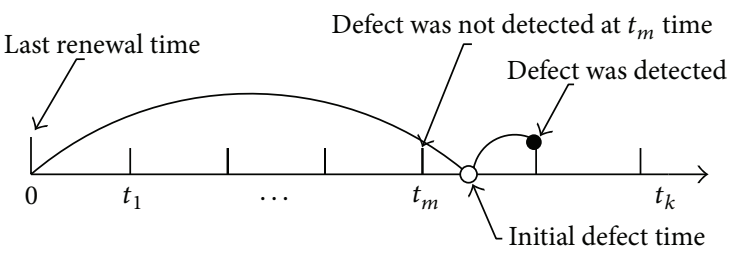

(b) Inspection renewal event

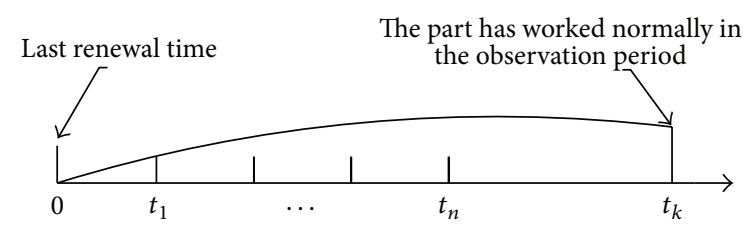

(c) Nonrenewal event

FIGURE 3: Function inspection process.

inspection renewal event, and a nonrenewal event, as shown in Figure 3 [12].

We combine the function inspection process of the wearing parts above with the theory of the DTM [2] to set up the failure density function or the failure probability function of the three kinds of events [20].

Let $X$ be a random variable of the time to failure. Then, the probability of the $i$ th inspection renewal happening at time $t_{i}$ is given by

$$
\begin{aligned}
P_{m}\left(t_{i}\right) & =P\left(X<t_{i}\right) \\
& =\int_{t_{m}}^{t_{i}} g(u)\left[1-F\left(t_{i}-u\right)\right] d_{u},
\end{aligned}
$$

where $P\left(X<t_{i}\right)$ is the probability that the time to failure $X$ is less than the inspection renewal time $t_{i}$.

The density function of the $j$ th failure renewal happens at time $t_{j}$, which is given by

$$
\begin{aligned}
p_{b}\left(t_{j}\right) & =\frac{d}{d t} P\left(X \leq t_{j}\right) \\
& =\int_{t_{n}}^{t_{j}} g(u) f\left(t_{j}-u\right) d_{u},
\end{aligned}
$$

where $P\left(X<t_{j}\right)$ is the probability that the time to failure $X$ is less than the failure renewal time $t_{j}$. 
The probability of no failure happening during the whole of observation period $t$ is given by

$$
\begin{aligned}
P_{n}(t) & =1-P(X<t) \\
& =1-\int_{0}^{t} g(u) d u+\int_{t_{k}}^{t} g(u)(1-F(t-u)) d_{u},
\end{aligned}
$$

where $P(X<t)$ is the probability that nonfailure time $X$ is less than the whole of observation period $t$.

3.3. The Nonideal Inspection Model. Some wearing parts have a complex structure and shape and therefore need greater inspection conditions and techniques in the inspection process. A few such defects cannot be identified perfectly, so we have assumed that the process of the function inspection for these kinds of wearing parts is a nonideal inspection case. Assuming that the probability of the defect being identified is $r$ and then referring to the ideal inspection model in Section 3.2 (including (1)-(3)), the density function or the probability function of the three kinds of events is established for the process of the nonideal inspection [20] and is given by

$$
\begin{aligned}
P_{m}\left(t_{i}\right)= & \sum_{j=1}^{m}(1-r)^{m-j+1} r \int_{t_{j-1}}^{t_{j}} g(u)\left[1-F\left(t_{i}-u\right)\right] d_{u} \\
& +r \int_{t_{m}}^{t_{i}} g(u)\left[1-F\left(t_{i}-u\right)\right] d_{u}, \\
P_{b}\left(t_{j}\right)= & \sum_{k=1}^{n}(1-r)^{n-k+1} \int_{t_{k-1}}^{t_{k}} g(u) f\left(t_{j}-u\right) d_{u} \\
& +\int_{t_{n}}^{t_{j}} g(u) f\left(t_{j}-u\right) d_{u}, \\
P_{n}(t)= & 1-\int_{0}^{t} g(u) d u+\int_{t_{k}}^{t} g(u)(1-F(t-u)) d_{u} \\
& +\sum_{j=1}^{k} \int_{t_{j-1}}^{t_{j}}(1-r)^{k-j+1} g(u)(1-F(t-u)) d_{u} .
\end{aligned}
$$

\section{Delay Time Model Algorithm}

4.1. Establishing the Likelihood Function. In view of the DTM characteristics of the two cases (ideal and nonideal inspection) and the solving method, we decided to use the maximum likelihood function method to estimate the parameters of the models. We take the density function or the probability function of the three kinds of renewal events for the wearing parts to establish the likelihood function of the DTM for a period of observed time [20], which is given by

$$
L(\Theta)=\prod_{i=1}^{N_{I}} P_{m}\left(t_{i}\right) \cdot \prod_{j=1}^{N_{F}} p_{b}\left(t_{j}\right) \cdot P_{n}(t),
$$

where $\Theta$ is the set of parameters of the probability density function and the cumulative distribution function for the initial time and delay time. The likelihood function is taken to look for the maximum, to obtain the estimated values of the parameters. This computing process can be simplified by taking natural logarithms to get the log-likelihood function:

$$
\begin{aligned}
\log L(\Theta)= & \sum_{i=1}^{N_{I}} \log \left[P_{m}\left(t_{i}\right)\right] \\
& +\sum_{j=1}^{N_{F}} \log \left[p_{b}\left(t_{j}\right)\right]+\log \left[P_{n}(t)\right] .
\end{aligned}
$$

Finally, the parameters of the DTM can be estimated by maximizing the log-likelihood.

4.2. Method of Solving Maximum Likelihood Function. The traditional maximum likelihood estimation (MLE) method used to adopt the derivative of likelihood function, solving its maximum value by setting the derivative equal to zero and finally solving the estimated parameters of the function. However, (6) is a more complicated log-likelihood function, containing the failure density function and the failure probability function with integral terms; taking the derivative of it is quite complex and cannot be solved by the traditional MLE method. Hence, for the parameter estimation, we need to find a suitable calculation method to cope with this problem. By analyzing the characteristics of (6) and the value range of the parameters in this study, we can convert it to an extremum problem of no constraints, which can be solved by sophisticated optimization algorithms. There are several nonderivative optimization algorithms: simplex substitution method (small-scale, rapid convergence), CRM (small-scale, noncoupling problem), grid point method (nonconvex problems), and Monte Carlo method (nonconvex problems) [21].

Since the log-likelihood function of (6) belongs to a small scale and noncoupling problem, we choose the CRM to estimate the parameters in the distribution function of the initial defect time and the defect delay time of wearing parts. The CRM is a direct search method without derivation: taking $n$ coordinate directions of $x$ as the search direction (namely, starting from $x^{0}$ ), the first search along the $x_{1}$ direction, the second search along the $x_{2}$ direction,..., the $n$th search along the $x_{n}$ direction, the $n+1$ th search along the $x_{1}$ direction, and so on, continuously search along the direction of rotation until the convergence condition is met [22].

The remaining calculation steps of the CRM are as follows.

Step 1. Select the initial point $x^{0}$ (also known as the initial base point), the initial step $\Delta x_{i}$, and the convergence precision $\varepsilon_{i}$.The initial step is generally a desirable variable estimation range $\left(u_{i}-l_{i}\right)$ of $1 / 100$, the convergence precision $\varepsilon_{i}$ can take the initial step $\Delta x_{i}$ of $1 / 100$, and let $k=0$.

Step 2. Begin a round of exploratory searching along itself direction one by one. To give $x_{1}$ an incremental $\Delta x_{1}$, other variables remain unchanged. Then we get a test point:

$$
x^{s}=\left(x_{1}^{(k+1)}+\Delta x_{1}, x_{2}^{(k+1)}, \ldots, x_{n}^{(k+1)}\right)^{T} .
$$


To examine the feasibility of $x^{s}$, if $f\left(x^{s}\right)<f\left(x^{k-1}\right)$, then $x^{s}$ begins as a coordinate direction search. Otherwise, search from point $x^{k-1}$ along the opposite direction:

$$
x^{s}=\left(x_{1}^{(k+1)}-\Delta x_{1}, x_{2}^{(k+1)}, \ldots, x_{n}^{(k+1)}\right)^{T} .
$$

If the two search directions fail, stopping at the original point and not moving, then take a step $\Delta x_{2}$ to search along the $x_{2}$ direction until $x_{n}$. The end point of this round of search was called base point $x_{B}^{k}$.

Step 3. Carry through the Pattern Move to point $x_{M}^{k}$. The Pattern Move direction is from a base point $x_{B}^{k-1}$ to the current base point $x_{B}^{k}$, and the amount of move is the distance between two points:

$$
x_{M}^{k}=x_{B}^{k}+\left(x_{B}^{k}-x_{B}^{k-1}\right) .
$$

Then, examine the feasibility of the Pattern Move. If $f\left(x_{M}^{k}\right)<f\left(x_{B}^{k}\right)$ is established, then take the point $x_{M}^{s}$ as the starting point for the next round search; otherwise, cancel this Pattern Move and take the point $x_{B}^{k}$ as the starting point for the next round search.

Step 4. Repeat Steps 2 and 3 to search. The Pattern Move is a loop execution until the exploratory searches of each coordinate direction have all failed and still remain unmoving in the original base point. Then, test whether it satisfies the convergence criteria $\Delta x_{i}<\varepsilon_{i}$ or not. If it is not satisfied, the variable step size $\Delta x_{i}(i=1,2,3, \ldots, n)$ is reduced by half, and a new round of exploratory search is started. If it is satisfied, put out an optimal point $x^{*}=x_{B}^{k}$ and $f\left(x^{*}\right)=f\left(x_{B}^{k}\right) \rightarrow$ $\min f(x)$.

\section{Numerical Example}

5.1. The Ideal Inspection Model Numerical Example. The filter element that plays a key role in the hydraulic system of the remote control station is a wearing part in the oil and gas drilling process. It has the advantages of a simple structure and low inspection requirements and is therefore considered as suitable for the ideal inspection model. Learning from the oil field maintenance records, the maintenance personnel renew the filter element for the hydraulic system in the following two cases.

(i) Failure renewal: if the filter element is clogged or leaking or has exploded and so forth, resulting in the well control equipment being unable to be operated in its normal state, we regard it as a failure and it will be maintained or replaced immediately.

(ii) Inspection renewal: within the regular inspection, the filter element needs to be cleaned or replaced when a large amount of grease and deposit has been detected in it.

Combined with the actual environments and conditions in the oilfield exploitation and construction, we found that
TABLE 1: Failure renewal time of the filter element.

\begin{tabular}{lccc}
\hline S/N & $\begin{array}{c}\text { Renewal time } \\
\text { (weeks) }\end{array}$ & Time & $\begin{array}{c}\text { Renewal time } \\
\text { (weeks) }\end{array}$ \\
\hline 1 & 21 & 16 & 30 \\
2 & 5 & 17 & 14 \\
3 & 22 & 18 & 33 \\
4 & 11 & 19 & 27 \\
5 & 15 & 20 & 8 \\
6 & 37 & 21 & 7 \\
7 & 26 & 22 & 25 \\
8 & 19 & 23 & 13 \\
9 & 18 & 24 & 29 \\
10 & 3 & 25 & 9 \\
11 & 23 & 26 & 11 \\
12 & 16 & 27 & 17 \\
13 & 10 & 28 & 15 \\
14 & 9 & 29 & 6 \\
15 & 18 & & \\
\hline
\end{tabular}

TABLE 2: Inspection renewal time of the filter element.

\begin{tabular}{lccc}
\hline S/N & Renewal time (weeks) & Time & Renewal time (weeks) \\
\hline 1 & 16 & 10 & 28 \\
2 & 4 & 11 & 12 \\
3 & 16 & 12 & 20 \\
4 & 20 & 13 & 16 \\
5 & 16 & 14 & 8 \\
6 & 32 & 15 & 28 \\
7 & 24 & 16 & 12 \\
8 & 32 & 17 & 36 \\
9 & 16 & 18 & 24 \\
\hline
\end{tabular}

the regular inspection interval for the filter element was approximately once every four weeks. Between 2003 and 2013, the filter element in a hydraulic system of a remote control station (FKQ-400-5-B) in the Tarim Oilfield had 29 failure renewals and 18 inspection renewals. Detailed maintenance records for the filter element were processed and collated, as shown in Tables 1 and 2.

Through analysis of the time distribution regularities of failure renewal and inspection renewal in Tables 1 and 2, we assumed that the initial time and the delay time follow the Weibull distribution or the exponential distribution. By using the data in Tables 1 and 2 with (1)-(3), we took those obtained equations into (5) to get the likelihood function of the DTM in an ideal inspection case. We then took the logarithm for it, to easily seek for the parameter estimation of the model. We considered that the maximum of the likelihood function was a small scale in the absence of a constraint range and took the CRM. The parameters of the initial time and the delay time of the filter element were estimated with different distribution types, as shown in Table 3. 
TABLE 3: The parameters estimation of the DTM for the filter element.

\begin{tabular}{|c|c|c|c|c|c|}
\hline \multirow{2}{*}{$\begin{array}{l}\text { Model } \\
\text { Parameter }\end{array}$} & \multicolumn{2}{|c|}{ The initial time $u$} & \multicolumn{2}{|c|}{ The delay time $v$} & \multirow[b]{2}{*}{$\mathrm{AIC}=2 \log L+2 k$} \\
\hline & $\alpha_{1}$ & $\beta_{1}$ & $\alpha_{2}$ & $\beta_{2}$ & \\
\hline$E-E$ & - & 17.98 & - & 2.36 & 56.24 \\
\hline$E-W$ & - & 40.42 & 1.23 & 5.94 & 62.08 \\
\hline$W-W$ & 1.23 & 18.24 & 0.27 & 2.09 & 60.16 \\
\hline$W-E$ & 1.12 & 18.06 & - & 2.21 & 58.84 \\
\hline
\end{tabular}

Notes: $W$ : Weibull distribution, $E$ : exponential distribution.

$W / E$ indicates that the initial time obeys a Weibull distribution, and the delay time is an exponential distribution.

"-_" means the parameters do not need to be estimated in the exponential distribution.

By comparing and analyzing the minimum of the Akaike information criterion (AIC), we find that the distribution regularities of the initial failure time and the delay time about the filter element obeying the exponential distribution are more accurate in the ideal inspection case [20]. From the results of Table 3, we have obtained their parameter estimation values: the average initial time $\bar{u}$ is 17.98 weeks, and the average delay time $\bar{v}$ is 2.36 weeks.

Based on the functional expression of the exponential distribution, we obtain the failure time distribution functions for the filter element: the probability density function of the initial failure time $g(u)=17.98 e^{-17.98 u}$, the cumulative distribution function of the initial failure time $G(u)=1-$ $e^{-17.98 u}$, the probability density function of the delay time $f(v)=2.36 e^{-2.36 v}$, and the cumulative distribution function of the delay time $F(v)=1-e^{-2.36 v}$.

5.2. The Nonideal Inspection Model Numerical Example. The Ram blowout preventer (Ram BOP) is one of the key safety devices in the well control system, whose main role is to ensure the safety of the drilling operation. Therefore, the field staff used to carry out a strict function inspection of it. The rubber seal on the ram end of the Ram BOP is called the rubber core, and it is an important component part that is the key to effectively closing the well in the process of well control. The rubber core is a wearing part with a very high failure frequency, and it used to be renewed in the following circumstances [23].

(i) Failure renewal: in the oil and gas well, the high pressure and high speed multiphase fluid that flows through the Ram BOP can corrode the rubber core, causing shedding or serious deformation in the process of closing the well, resulting in well liquid leakage and ineffective closing of the wellhead.

(ii) Inspection renewal: through regular inspection, if the rubber core is found to be forming an axial scratch, aging, corroding, cracking, or peeling, we need to replace it immediately.

(iii) Opportunity renewal: no matter how well the rubber core works, when we maintain the other parts of the Ram BOP, we also need to inspect the rubber core. If problems are found or potential failure is realized, it must be replaced immediately.
TABLE 4: Failure renewal time of rubber core.

\begin{tabular}{lcc}
\hline S/N & Failure renewal time (months) & $\begin{array}{c}\text { Early inspection time } \\
\text { (months) }\end{array}$ \\
\hline 1 & 3 & - \\
2 & 7 & - \\
3 & 12 & 4 \\
4 & 4 & - \\
5 & 15 & 7 \\
6 & 23 & 3,12 \\
7 & 19 & 5,13 \\
8 & 5 & - \\
9 & 15 & 8 \\
10 & 1 & - \\
\hline
\end{tabular}

TABLE 5: Inspection renewal time of the rubber core.

\begin{tabular}{lcc}
\hline S/N & Failure renewal time (months) & $\begin{array}{c}\text { Early inspection time } \\
\text { (months) }\end{array}$ \\
\hline 1 & 2 & - \\
2 & 16 & 8 \\
3 & 18 & 10 \\
4 & 5 & - \\
5 & 10 & - \\
6 & 12 & 2,8 \\
7 & 9 & 6 \\
8 & 8 & 2 \\
\hline
\end{tabular}

In drilling operation, it is necessary to carry out a function inspection for the Ram BOP approximately every 1-2 months. After the completion of the drilling, the Ram BOP is sent back to the equipment factory approximately every 5-10 months for maintenance. Based on the actual situation, the rubber core of the Ram BOP meets a nonideal process of function inspection, where the probability of perfectly inspecting the defect (through the consultation of on-site experts) generally ranges from $80 \%$ to $90 \%$. Through research into the Tarim Oilfield, we have the repair record of the rubber core in the Ram BOP (FZ35-70), as shown in Tables 4, 5, and 6. 
TABLE 6: Opportunity renewal time of the rubber core.

\begin{tabular}{lcc}
\hline S/N & $\begin{array}{c}\text { Opportunity renewal time } \\
\text { (months) }\end{array}$ & $\begin{array}{c}\text { Early inspection time } \\
\text { (months) }\end{array}$ \\
\hline 1 & 6 & - \\
2 & 11 & 4 \\
3 & 12 & - \\
4 & 4 & - \\
5 & 12 & 7 \\
6 & 9 & - \\
7 & 15 & 8 \\
\hline
\end{tabular}

Because of the existence of the opportunistic repair of the rubber core in the function inspection process of the Ram BOP, the maximum likelihood function was changed [20]:

$$
L=\prod_{i=1}^{N_{I}} P_{m}\left(t_{i}\right) \cdot \prod_{j=1}^{N_{F}} p_{b}\left(t_{j}\right) \cdot \prod_{c=1}^{N_{c}} P_{n}\left(t_{c}\right) .
$$

We can assume that the initial time and the delay time follow the Weibull distribution or the exponential distribution. By using the data in Tables 4-6 with (4), we took those obtained equations into (10) to get the likelihood function of the DTM in a nonideal inspection case.

We then took the logarithm for it to get the log-likelihood, to seek for reasonable parameters of the DTM by the maximum likelihood in the no constraint range. Finally, through calculation with the CRM, the initial time and delay time parameters of the model of the rubber core can be estimated, as shown in Table 7.

From the parameter estimation results in Table 7, we can see that both the initial time and the delay time of rubber core obeying the Weibull distribution are more accurate because of the value of AIC minimum, and the most reasonable values of parameter estimation are $\alpha_{1}=1.25, \beta_{1}=10.28 ; \alpha_{2}=2.21$, $\beta_{2}=3.27$. Therefore, the average initial time $\bar{u}$ is 10.28 months and the average delay time $\bar{v}$ is 3.27 months.

Based on the functional expression of the Weibull distribution, we obtain the probability density function of the initial failure time as

$$
g(u)=0.12\left(\frac{u}{10.28}\right)^{0.25} e^{-(u / 10.28)^{1.25}}
$$

The cumulative distribution function of the initial failure time is

$$
G(u)=1-e^{(u / 10.28)^{1.25}} .
$$

The probability density function of the delay time is

$$
f(v)=0.68\left(\frac{u}{3.27}\right)^{1.21} e^{-(u / 3.27)^{2.21}}
$$

The cumulative distribution function of the delay time is

$$
F(v)=1-e^{(v / 3.27)^{2.21}}
$$

As we all know, PM includes a regular inspection, repair, and replacement routine designed to reduce the risk of system failure. The probability density function and the cumulative distribution function of failure time, including the initial time and delay time, play a very important role in the application of the DTM, and the accurate parameter estimation for the model also directly affects the optimization of the reasonable PM interval. Therefore, we have obtained an optimal value of parameter estimation for the initial time model and delay time model based on the objective data, and these models can be applied to the function test model of the potential unmeasured failures to optimize the PM interval on account of the cost, availability, and risk guideline.

\section{Conclusion}

In the ideal inspection and nonideal inspection cases, the DTM (including the failure renewal model, inspection renewal model, and nonfailure renewal model) was established for the wearing parts, which can be extended to other single components and simple systems obtaining a large number of objective maintenance data. Moreover, the maximum likelihood for DTM was solved by the CRM in the no constraint range. The examples of the filter element for the hydraulic system of a remote control station and the rubber core of Ram BOP were verified for the ideal inspection model and nonideal inspection model, respectively. We have estimated the parameters of the DTM and determined more accurate distribution types for their failure function, and both the average initial defect time and the average failure delay time were determined to provide data for the further function to optimize the inspection interval, which verifies the feasibility of the models and the method. We then obtained the probability density function and the cumulative distribution function of failure time, including the initial time and the delay time. This study provides a specific DTM for the wearing parts, which will help to provide an optimal function inspection interval, lower repair costs, and establish a maintenance decision model to reduce the risk of system failure.

\section{Conflict of Interests}

The authors declare that there is no conflict of interests regarding the publication of this paper.

\section{Acknowledgments}

The work received the support of the Natural Science Foundation of China (Grant no. 51274171) and the Graduate Student 
TABLE 7: The parameter estimation of the DTM for the rubber core.

\begin{tabular}{lcccccc}
\hline Model & \multicolumn{2}{c}{ The initial time $u$} & \multicolumn{2}{c}{ The delay time $v$} & \\
\hline Parameter & $\alpha_{1}$ & $\beta_{1}$ & $\alpha_{2}$ & $\beta_{2}$ & & AIC $=2 \log L+2 k$ \\
\hline$E-E$ & - & 12.56 & - & 5.21 & 0.80 & 39.04 \\
$E-W$ & - & 10.83 & 0.72 & 5.42 & 0.80 & 40.48 \\
$W-W$ & 1.25 & 10.28 & 2.21 & 3.27 & 0.80 & 38.72 \\
$W-E$ & 1.50 & 10.02 & - & 5.05 & 0.80 & 40.16 \\
\hline
\end{tabular}

Notes: $W$ : Weibull distribution, $E$ : exponential distribution.

$W / E$ indicates that the initial time obeys a Weibull distribution, and the delay time is an exponential distribution.

"-_" means the parameters do not need to be estimated in the exponential distribution.

Innovation Fund of School of Mechatronic Engineering, Southwest Petroleum University (CX2014BZ04). Furthermore, the authors are grateful to the Safety, Environment, Quality Supervision \& Testing Research Institute, CCDE.

\section{References}

[1] M. Rausand, "Reliability centered maintenance," Reliability Engineering and System Safety, vol. 60, no. 2, pp. 121-132, 1998.

[2] W. Wang, "An overview of the recent advances in delay-timebased maintenance modelling," Reliability Engineering and System Safety, vol. 106, pp. 165-178, 2012.

[3] A. H. Christer, "Delay-time model of reliability of equipment subject to inspection monitoring," Journal of the Operational Research Society, vol. 38, no. 4, pp. 329-334, 1987.

[4] A. H. Christer and W. M. Waller, "Delay time models of industrial inspection maintenance problems," Journal of the Operational Research Society, vol. 35, no. 5, pp. 401-406, 1984.

[5] A. H. Christer and D. F. Redmond, "A recent mathematical development in maintenance theory," IMA Journal of Mathematics, vol. 2, no. 2, pp. 97-108, 1989.

[6] R. D. Baker and W. Wang, "Estimating the delay-time distribution of faults in repairable machinery from failure data," IMA Journal of Management Mathematics, vol. 3, no. 4, pp. 259-281, 1991.

[7] R. D. Baker and W. Wang, "Developing and testing the delaytime model," Journal of the Operational Research Society, vol. 44, no. 4, pp. 361-374, 1993.

[8] A. H. Christer and W. Wang, "A delay-time-based maintenance model of a multi-component system," IMA Journal of Management Mathematics, vol. 6, no. 2, pp. 205-222, 1995.

[9] D. Wang, L. Wen, and X. S. Jia, "Maximum likelihood estimation of delay time mode," Journal of Ordnance Engineering College, vol. 17, no. 3, pp. 33-35, 2005.

[10] H. Hu, G. Cheng, Q. Duan, W. Wu, and C. Xu, "Delay time model based on imperfect maintenance," Journal of Xian Jiaotong University, vol. 43, no. 6, pp. 103-107, 2009.

[11] W. Wang, "A two-stage prognosis model in condition based maintenance," European Journal of Operational Research, vol. 182, no. 3, pp. 1177-1187, 2007.

[12] W. Wang, "An inspection model based on a three-stage failure process," Reliability Engineering and System Safety, vol. 96, no. 7, pp. 838-848, 2011.

[13] W. Wang and X. S. Jia, "A Bayesian approach in delay time maintenance model parameters estimation using both subjective and objective data," Quality Maintenance and Reliability Int, vol. 23, no. 3, pp. 95-105, 2007.
[14] S. Apeland and P. A. Scarf, "A fully subjective approach to modelling inspection maintenance," European Journal of Operational Research, vol. 148, no. 2, pp. 410-425, 2003.

[15] W. Wang, "An inspection model for a process with two types of inspections and repairs," Reliability Engineering and System Safety, vol. 94, no. 2, pp. 526-533, 2009.

[16] A. H. Christer, W. Wang, J. Sharp, and R. Baker, "A case study of modelling preventive maintenance of a production plant using subjective data," Journal of the Operational Research Society, vol. 49, no. 3, pp. 210-219, 1998.

[17] W. G. Aiello and H. I. Freedman, "A time-delay model of singlespecies growth with stage structure," Mathematical Biosciences, vol. 101, no. 2, pp. 139-153, 1990.

[18] A. H. Christer, C. Lee, and W. Wang, "Data deficiency based parameter estimating problem and case study in delay time PM modeling," International Journal of Production Economics, vol. 67, no. 1, pp. 63-76, 2000.

[19] T. Aven and I. T. Castro, "A delay-time model with safety constraint," Reliability Engineering and System Safety, vol. 94, no. 2, pp. 261-267, 2009.

[20] X. S. Jia, The Decision Models for Reliability Centered Maintenance, National Defense Industry Press, 2007.

[21] R. D. Baker, P. A. Scarf, and W. Wang, "A delay-time model for repairable machinery: maximum likelihood estimation of optimum inspection intervals," IMA Journal of Management Mathematics, vol. 8, no. 1, pp. 83-92, 1997.

[22] Z. Bačić and J. Simons, "Complex coordinate rotation calculation of branching ratios," International Journal of Quantum Chemistry, vol. 21, no. 4, pp. 727-739, 1982.

[23] A. H. Christer, W. Wang, R. D. Baker, and J. Sharp, "Modelling maintenance practice of production plant using the delay-time concept," IMA Journal of Management Mathematics, vol. 6, no. 1, pp. 67-83, 1995. 


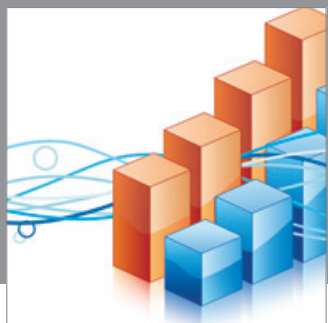

Advances in

Operations Research

mansans

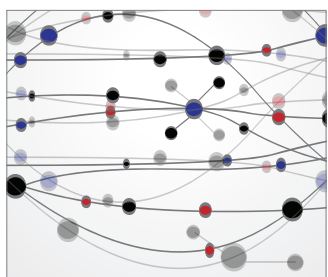

The Scientific World Journal
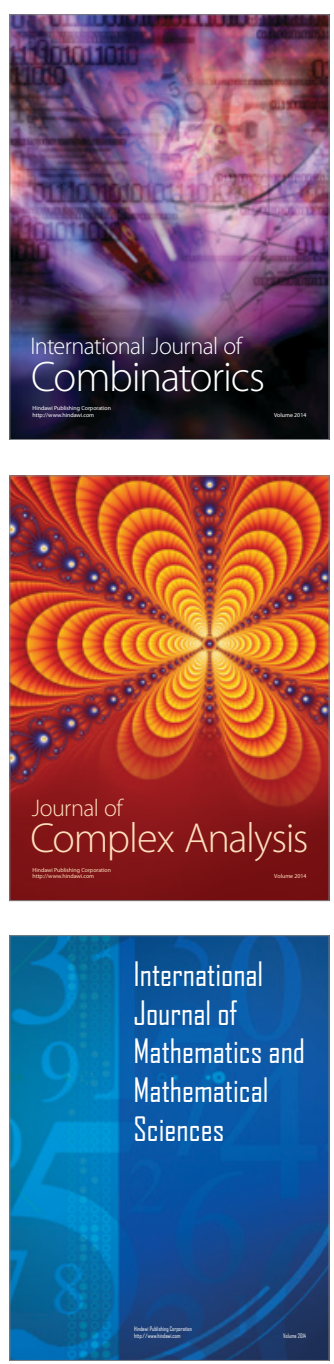
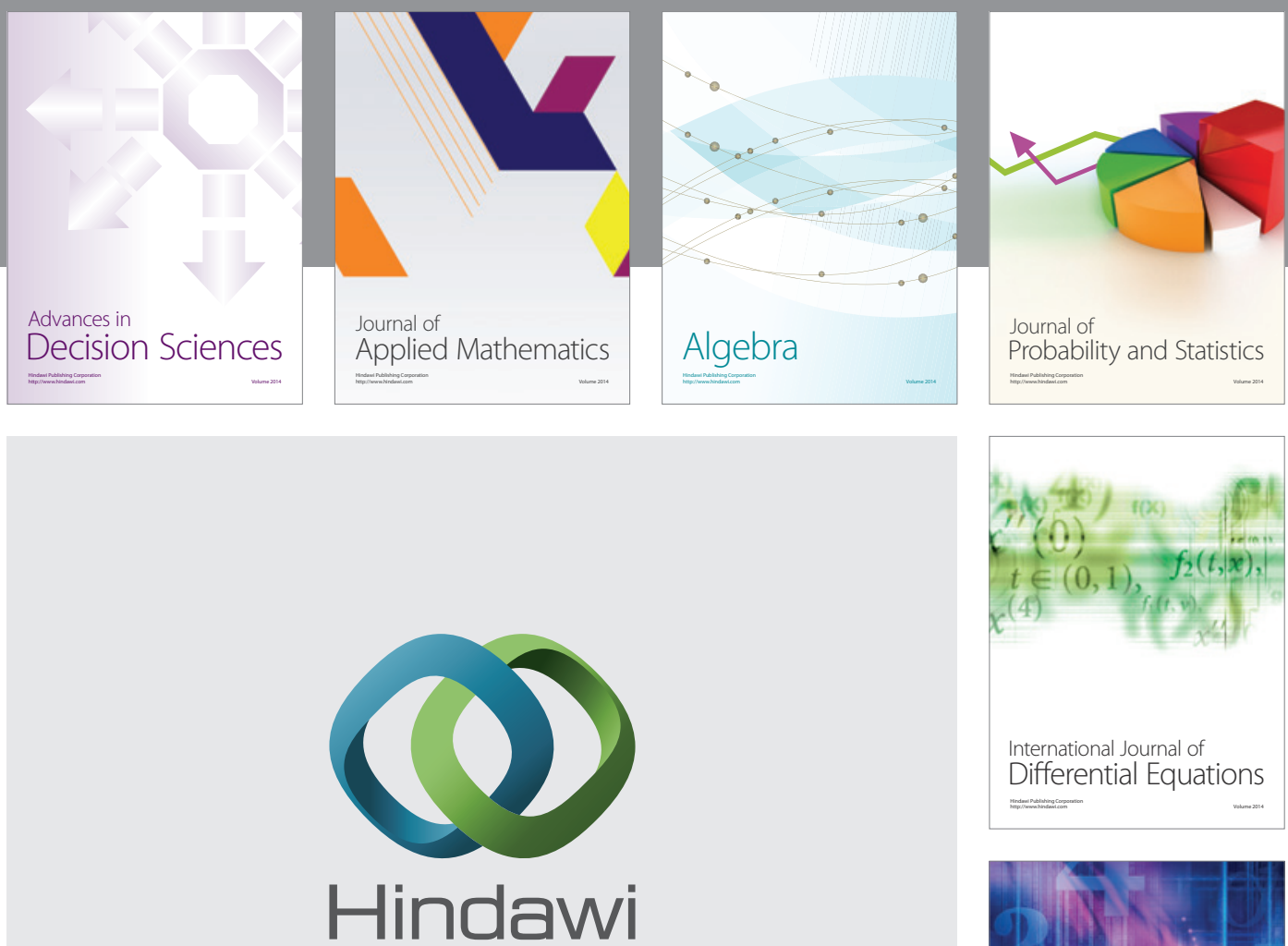

Submit your manuscripts at http://www.hindawi.com
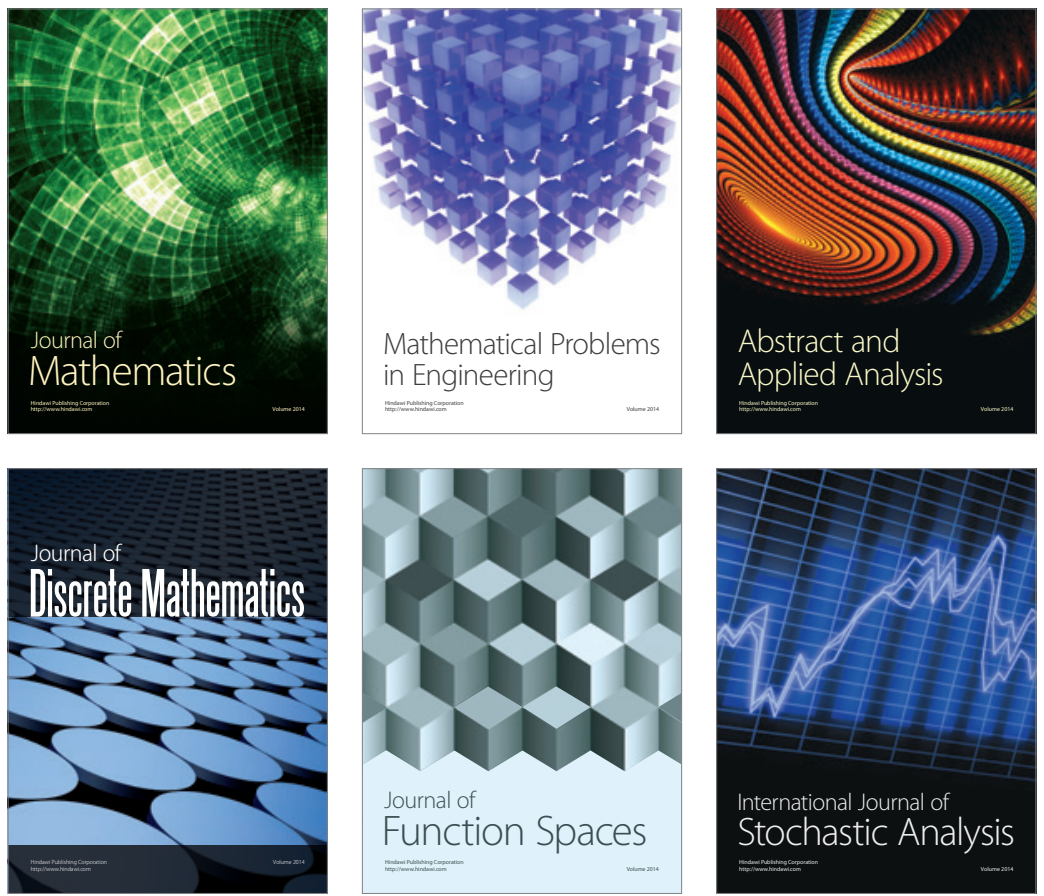

Journal of

Function Spaces

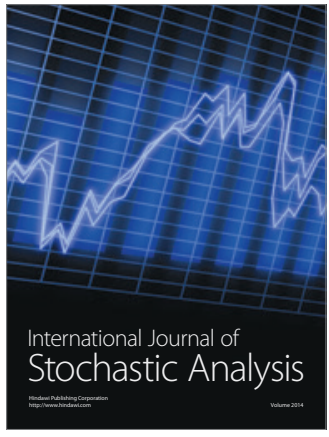

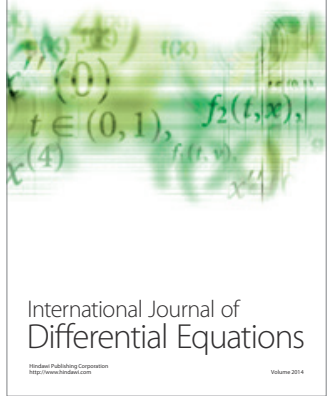
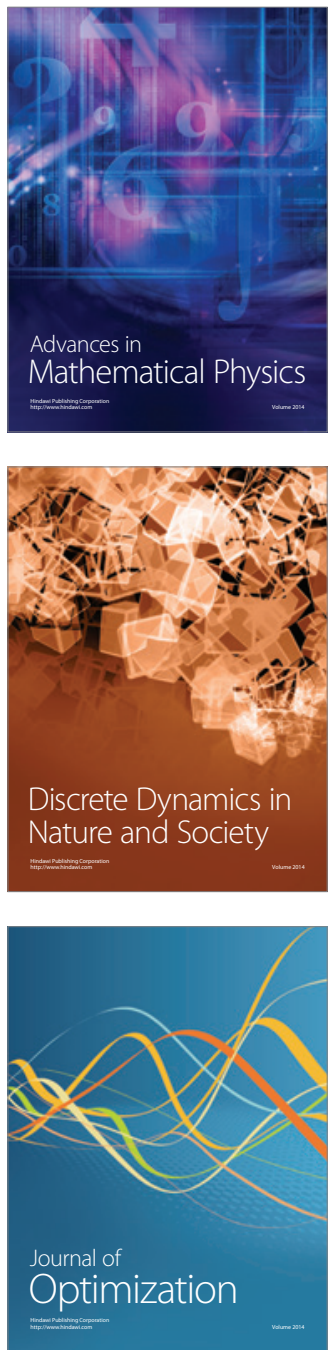